\title{
A FINE-TURNING COPOLYMER BASED ON TRIPHENYLAMINE AND 3,4-ETHYLENEDIOXYTHIOPHENE VIA ELECTROCHEMICAL POLYMERIZATION
}

\author{
MI OUYANG \\ State Key Laboratory Breeding Base for Green Chemistry Synthesis Technology, \\ Zhejiang University of Technology, Hangzhou, 310014, PR China \\ ouyang@zjut.edu.cn, \\ BIN HU \\ State Key Laboratory Breeding Base for Green Chemistry Synthesis Technology, \\ Zhejiang University of Technology, Hangzhou, 310014, PR China \\ hubin30003@163.com \\ CHENG ZHANG \\ State Key Laboratory Breeding Base for Green Chemistry Synthesis Technology, \\ Zhejiang University of Technology, Hangzhou, 310014, PR China \\ czhang@zjut.edu.cn
}

\begin{abstract}
Copolymer based on triphenylamine (TPA) and 3,4-ethylenedioxythiophene (EDOT) is polymerized electrochemically. The structure, morphology and electrochemical properties of the obtained copolymer are investigated by cyclic voltammetry and FT-IR spectroscopy. The results confirm the copolymerization of TPA/EDOT. UV-vis spectra show that the absorption peaks of the copolymer are different from those of Polytriphenylamine. Moreover, photoluminescence spectra of the copolymer exhibit great changes in emission color from pale blue to pale green with the variety of ratio of two monomers. Therefore, the monomer feed ratio plays important roles in the optical property of this copolymer.
\end{abstract}

Keywords : Copolymer; Electrochemical polymerization; Optical property

\section{Introduction}

Since the first discovery of polymer-based light-emitting diodes (PLEDs) in $1990^{1}$, considerable progress has been made in the development of new conjugated polymers and exploration of their applications in displays ${ }^{2,3}$. The synthesis methods of these materials can be carried out by either chemical or electrochemical polymerization. Compared with the chemical route, electrochemical polymerization can obtain conjugated polymer films on conductive substrate directly. This not only enlarges the scope of candidate polymers ${ }^{4}$, but also omits the procedure of the film coating ${ }^{5}$. 
As we all know, triphenylamine (TPA) has been widely used as optical and electroactive materials for its good electron donating and transporting capability ${ }^{6,7}$. However, few report is about its copolymer with other materials by electrochemical polymerization. The reason may be that TPA could be easily dimerized to form tetraphenylbenzidine, but incorporating electron-donating groups can prevent such a phenomenon $^{8}$. EDOT is a common monomer to be used as donated group which can present outstanding electrochemical stability and activity ${ }^{9,10}$. It can be expected that incorporation of EDOT unit into PTPA may show some special properties.

In this paper, EDOT is successfully introduced into PTPA main-chains via the electrochemical oxidation of the TPA/EDOT mixtures. The electrochemical performances and the optical properties of PTPA, PEDOT and TPA/EDOT copolymers are investigated in detail. The synthetic schemes of the copolymer are illustrated in scheme 1.

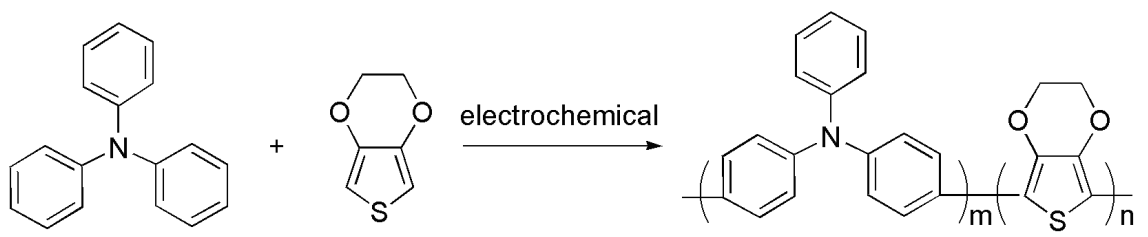

Scheme 1. Electrochemical polymerization route of TPA and EDOT.

\section{Experimental}

\subsection{Raw materials}

All regents are commercial products which purchase from Aladdin Scientific .

\subsection{Electrochemical polymerization}

Electrocopolymerization of TPA and EDOT were performed in an Acetonitrile(ACN) solution containing $0.1 \mathrm{M}$ Tetrabutylammonium perchlorate (TBAP). Indium-tin oxide (ITO) was used as working electrode, a platinum (Pt) sheet was used as counter electrode and $\mathrm{Ag} / \mathrm{AgCl}$ in saturated $\mathrm{KCl}$ (aq) solution as reference electrode. The films for charactered were prepared via potentiostatic electrolysis on ITO electrodes in the solutions of $0.1 \mathrm{M}$ TPA and $0.1 \mathrm{M}$ EDOT, $0.1 \mathrm{M}$ TPA and $0.2 \mathrm{M}$ EDOT or $0.1 \mathrm{M}$ TPA and $0.3 \mathrm{M}$ EDOT at room temperature, then the films were electrolyzed at negative potential for several minutes in monomer-free electrolytic solution, then the films were washed with clean $\mathrm{ACN}$ for several times to remove the residual supporting electrolyte and the monomers 


\subsection{Characterizations}

Electrochemical measurements were performed on a CHI 660C. Infrared spectra were recorded using a Thermo Nicolet 6700 FT-IR spectrometer. UV-vis spectra were carried out on a Varian Cary 100 UV-vis spectrophotometer. Thermal analysis were done by a Diamond TG/DTA 6300.

\section{Results and Discussion}

\subsection{Electro-copolymerization of TPA and EDOT}

The continuous CV curves of TPA, TPA/EDOT and EDOT are illustrated in Fig. 1. Though polymerization curves of TPA present an oxidation peak and a reduction peak (Fig. 1a), the CV of the first circle and the fifth circle show no obvious change, which indicates that the dimer is formed ${ }^{11}$, but it is not stable and prone to dissolve in ACN. While the polymerization curves of EDOT (Fig.1c) reveal an oxidation peak at $+1.23 \mathrm{~V}$ and a reduction peak at $-0.42 \mathrm{~V}$. In contrast, the redox behavior of the TPA/EDOT mixture (oxidation peak: $+1.02 \mathrm{~V}$ and reduction peak: $+0.53 \mathrm{~V}$, Fig. $1 \mathrm{~b}$ ) is obviously different from those of PTPA and PEDOT, indicating the occurrence of copolymerization and the formation of a copolymer containing TPA and EDOT units

\subsection{Structural characterizations}

The FT-IR spectra of PEDOT, PTPA and PolyTPA/EDOT are shown in Fig. 2. (PTPA is gained by chemical polymerization ${ }^{12}$ ). According to the spectrum of PEDOT, the bands at 1513,1322 and $1195 \mathrm{~cm}^{-1}$ originate from the stretching modes of $\mathrm{C}=\mathrm{C}$ and $\mathrm{C}-\mathrm{C}$ in the thiophene rings (Fig.2a), the bands at 1084 and $980 \mathrm{~cm}^{-1}$ arise from the stretching modes of C-O-C and C-S-C in EDOT rings, respectively.

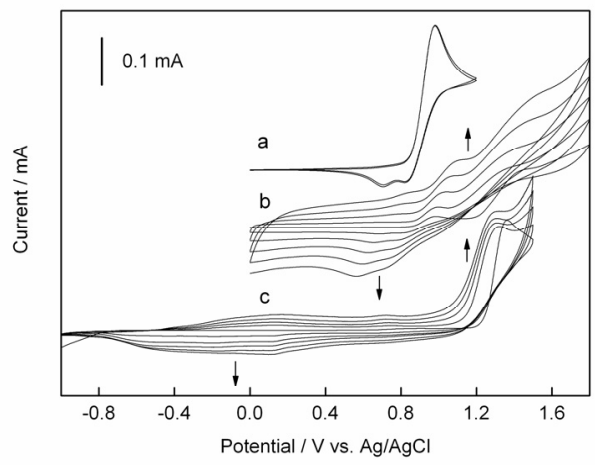

Fig. 1. The CV curves of (a) $0.01 \mathrm{M}$ TPA, (b) $0.01 \mathrm{M}$ TPA $+0.01 \mathrm{M}$ EDOT and (c) $0.01 \mathrm{M}$ EDOT in $0.1 \mathrm{M}$ TBAP/ACN.

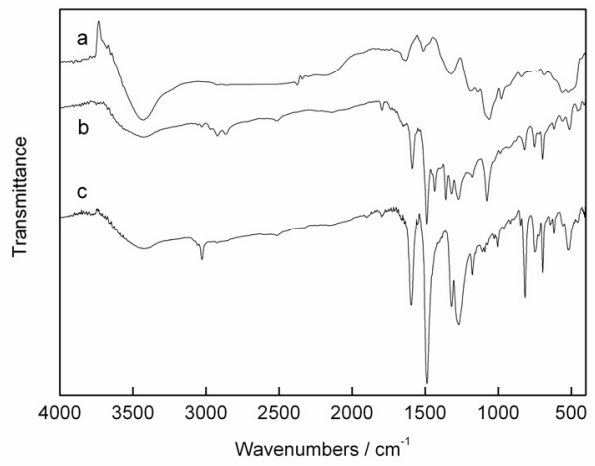

Fig. 2. FT-IR curves of (a) PEDOT, (b)P(TPA/EDOT) and (c) PTPA.

In the spectrum of PTPA, the peaks at 3057 and $3027 \mathrm{~cm}^{-1}$ reflect $\mathrm{C}-\mathrm{H}$ of the benzene rings, the bands at 1595 and $1487 \mathrm{~cm}^{-1}$ are originated from the stretching modes 
of $\mathrm{C}-\mathrm{C}$ of the benzene rings, the bands at 1319 and $1269 \mathrm{~cm}^{-1}$ are assigned to the stretching modes of N-C of TPA and the bands at 750 and $695 \mathrm{~cm}^{-1}$ demonstrate the benzene rings that don't participate in reaction, indicating not all the benzene rings of TPA participate in the reaction(Fig.2c). Compared with PTPA, Poly(TPA/EDOT) (Fig. 2b) exhibits bands at 1595,1487 and $1319 \mathrm{~cm}^{-1}$, indicating the presence of TPA units. The bands at 1357 and $1088 \mathrm{~cm}^{-1}$, originated from EDOT rings, can also be found. All the above features indicate that P(TPA/EDOT) contains both TPA and EDOT units.

\subsection{Optical properties}

The UV-vis spectra of PTPA and copolymers with different feed ratios in DMSO solutions are shown in Fig. 3. All the spectra have a strong absorption peak at $380 \mathrm{~nm}$ (Fig. 3a), which is arised by TPA units. Compared with PTPA, the copolymer shows an obvious absorption peak between $400-500 \mathrm{~nm}$, which is caused by $\pi-\pi *$ in copolymer.

The fluorescence spectra of the copolymers solutions show red-shift emitting properties (Fig. 4). PTPA exhibits the emission peak at $430 \mathrm{~nm}$, and the copolymers with the feed ratio of TPA/EDOT at 1:1, 1:2, 1:3 show their emission peaks at 480, 525 and $540 \mathrm{~nm}$, respectively. The red-shift of emission peaks present that the bandgaps of copolymers decrease as the contents of EDOT increase in the obtained copolymers(Table.1).

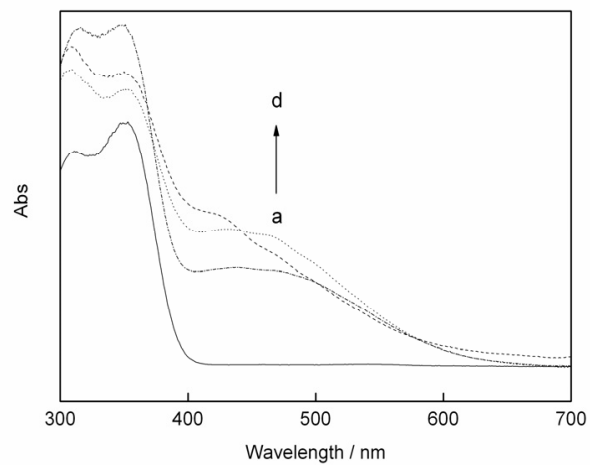

Fig. 3. UV-vis spectra of (a) PTPA, and copolymers with various feed of TPA/EDOT: (b) $1: 1$, (c) $1: 2$, (d) $1: 3$

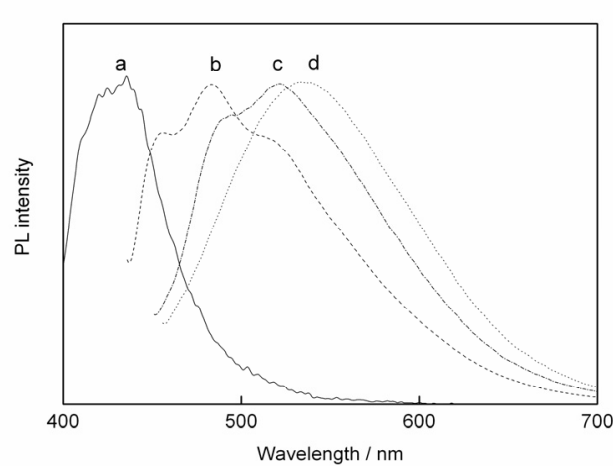

Fig. 4. PL spectra of (a) PTPA, and copolymers with various feed of TPA/EDOT:(b) $1: 1$, (c) 1:2, (d) $1: 3$.

Table 1. Max. wavelength of UV-vis spectra and PL and bandgap of copolymers with various feed ratios

\begin{tabular}{ccccc}
\hline Feed ratio of TPA/EDOT & $1: 0$ & $1: 1$ & $1: 2$ & $1: 3$ \\
\hline UV-vis (nm) & 350 & 350,460 & 350,460 & 350,460 \\
PL (nm) & 433 & 483 & 520 & 532 \\
Eg (eV) & 2.89 & 2.57 & 2.39 & 2.33 \\
\hline
\end{tabular}




\subsection{Thermal analysis}

The thermal stability of a conjugated polymer is very important for its potential application in the fields of PLEDs and PSCs. The thermogravimetry (TG) curves of PTPA, P(TPA/EDOT) and PEDOT are shown in Fig. 5. It's clear that copolymer presents the onset temperature of weight loss at about $300{ }^{\circ} \mathrm{C}$, which is higher than that of PEDOT at about $200{ }^{\circ} \mathrm{C}$.

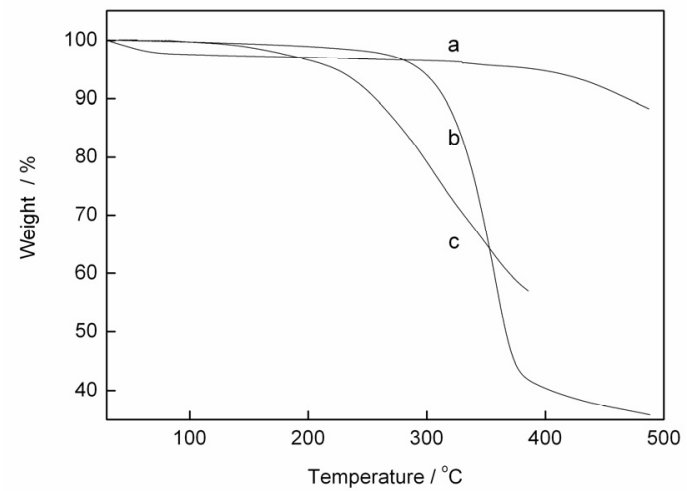

Fig. 5. TG curves of (a) PTPA, (b) Poly(TPA/EDOT) and (c) PEDOT.

\section{Conclusions}

The copolymers containing the moieties of EDOT and TPA have been successfully prepared via electrochemical copolymerization. The fluorescence spectra of the copolymers with different feed ratios show red-shift with the maximum wavelength from $430 \mathrm{~nm}$ to $540 \mathrm{~nm}$. The results of TG show the copolymer has good thermal stability. This fine-turning polymer can be a promising candidate material for PLED devices.

\section{Acknowledgment}

This work is financially supported by National Basic Research Program of China (2010CB635108, 2011CBA00700), Natural Science Foundation of Zhejiang Province, China (Y4090260), Major Science and Technology, Special and Priority Themes of Zhejiang Province, China (2009C14004)

\section{References}

1. J.H. Burroughes, D.D.C. Bradley, A.R. Brown, R.N. Marks, K. Mackay, R.H. Friend, P.L. Burns, A.B. Holmes, Nature. 347, 539(1990)..

2. M.T. Bernius, M. Inbasekaran, J. O’Brien, W. Wu,. Adv. Mater. 12, 1737(2000).

3. J. Li, Z.S. Bo. Macromolecules 37, 2013(2004).

4. C.C. Chang, L.J. Her, J.L. Hong, Electrochim. Acta. 50, 4461(2005).

5. Balan, D. Baran, L. Toppare, J. Mater. Chem. 20, 9861(2010).

6. S. Roquet, A. Cravino, P. Leriche, O. Aleveque, P. Frere, J. Roncali, J. Am. Chem. Soc., 128, 3459(2006). 
149 M. Quyang, B. Hu \& C. Zhang

7. X.M. Zhang, Y.W. Quan, Z. Cui, Q.M. Chen, J.F. Ding, J.P. Lu, Eur. J. Org. Chem. 2010, 2295(2010).

8. K.Y. Chiu, T.X. Su, J. H. Li, T. H. Lin, G.S. Liou, S.H. Cheng, J. Electroanal. Chem. 575, 95(2005),

9. G. Wang, X.K. Fu, J. Huang, L. Wu, Q.L. Du, Electrochim. Acta. 55, 6933(2010)

10. H. Zhang, Y. Wang, K. Shao, Y. Liu, S. Chen, W. Qiu, X. Sun, T. Qi, Y. Ma, G. Yu, Z. Su, D. Zhu, Chem. Commun. 755(2006).

11. A. Petr, C. Kvarnstrom, L. Dunsch, A. Ivaska, Synth. Met. 108(3): 245(2000).

12. C. Takahashi., S. Moriya, N. Fugono, H,C. Lee, H. Sato,. Synth. Met. 129(2): 123(2002). 